\title{
The molecular systemic and local effects of intra- tendinous injection of Platelet Rich Plasma in tendinosis: preliminary results on a rat model with ELISA method
}

\author{
Benjamin Dallaudiere ${ }^{1,2}$ \\ Liliane Louedec ${ }^{2}$ \\ Marie Paule Jacob Lenet ${ }^{2}$ \\ Lionel Pesquer ${ }^{2}$ \\ Elvind Blaise ${ }^{2}$ \\ Anne Perozziello² \\ Jean Baptiste Michel$^{2}$ \\ Maryse Moinard ${ }^{2}$ \\ Philippe Meyer ${ }^{2}$ \\ Jean Michel Serfaty² \\ 1 Centre d'Imagerie Ostéo-articulaire, Clinique du \\ Sport de Bordeaux-Mérignac, France \\ 2 Department of MSK Radiology Department, CHU \\ Pellegrin, Bordeaux, France
}

Corresponding author:

Benjamin Dallaudiere

Department of MSK Radiology Department, CHU Pellegrin

Place Amélie Léon Rabat

33000 Bordeaux, France

E-mail: benjamin.dallaudiere@gmail.com

\section{Summary}

Purpose: the aim of our study was thus to quantify the effect of Platelet Rich Plasma (PRP) injection on systemic and local growth factors and to identify molecular markers in a rat model of patellar and Achilles tendinosis treated with PRP.

Material and method: twenty two rats were used for the study. Two healthy rats were used as control (T-). We induced tendinosis ( $T+)$ in 20 rats $(80$ tendons by injecting under ultrasonography (US) guidance Collagenase $1 \circledR$ (day $0=\mathrm{D} 0$, patellar $=40$ and Achilles=40).

At D3, these 20 rats with tendinosis were separated in treatment by either PRP (PRPT+, $n=28$ ), physiological serum (PST+, $n=28$, control) USguided intratendinous injection, or without no PRP or PS $(T+, n=24$, control of natural evolution of tendinopathy). Follow-up at D7, D13, D18 and D25 using serum sample and local tendon removal with ELISA technics and comparison between the 3 groups were performed.

Results: during biological follow up, comparison of all serum samples of PRPT+, PST+ and T+ groups showed no significant modification of their biological markers at D7, D13, D18 and D25 (p>0.22). Comparison of immunological sample tendon markers of PRPT+, PST+ and T+ groups also showed no significant modification of markers at D7, D13, D18 and D25 ( $p>0.16)$ considering each biological marker and also all subgroups confounded.

Conclusion: our study strongly suggests that a single intratendinous US-guided injection of PRP in Achilles and patellar T+ doesn't increase biological markers such as growth factors compared to a control group in mid-term and long-term follow-up.

KEY WORDS: tendinosis, rat, platelet, PRP, ELISA.

Key Points: our goal was to assess the systemic and local molecular effect of intratendinous injection of PRP in tendinosis.

We used patellar and Achilles tendinosis in a rat model with adequate controls.

We precisely defined platelet, leukocyte concentrations in PRP, on a large cohort.

We evaluated PRP biological molecular systemic and local effects.

We provide strong evidence that PRP didn't increase biological markers, particularly growth factors, in serum and tendon dosages in PRP treated tendinosis compared to placebo group in mid-term and longterm follow-up.

\section{Introduction}

Tendinosis $(T+)$ is a very common and disabling condition, resulting in impairment of quality of life. Indeed, $T+$ of the rotator cuff is the most common musculoskeletal cause of shoulder pain in the general population, mainly women between 40 and 65 year old in Europe whereas Achille's T+ affects $5-6 \%$ of the general population, especially young men in North America. In most cases, this condition progresses to a disabling pain or tendon rupture ${ }^{1,2}$.

The healthy tendon is composed of type 1 collagen and a few elastic fibers, within a ground substance containing cells (tenocytes and tenoblasts) and water. In case of $\mathrm{T}+$, histology mainly shows thinned and disorganized collagen fibers and increased interfibrillar glycosaminoglycans deposition with production of prostaglandins [PGE2, Interleukines (IL6, IL1B), cyclooxygenase (COX2) and matrix metalloproteinase 
(MMP1, MMP3) expression]. Neo-angiogenesis and nerve fiber development have also been reported at the beginning of $\mathrm{T}+$ and throughout tendon healing. Inflammatory lesions are rare, but may be associated with tendon rupture ${ }^{3}$. Early treatment of $T+$ should therefore be recommended to avoid complications. Several lines of research have been explored for the treatment of $\mathrm{T}+$ and tendon rupture, including Ultrasound (US)-guided fenestration or tenotomy ${ }^{4}$, and intratendinous injections of hyperosmolar solutions ${ }^{5}$, bone morphogenic protein 6 , or platelet-rich plasma (PRP), with varying efficiencies ${ }^{7,8}$. Despite these potential treatments, peri-tendinous injection of corticosteroid remains the commonly accepted strategy to treat diseases of the tendon, despite the absence of inflammation in $\mathrm{T}+$ in this condition, and the proven serious side effects (tendon rupture) ${ }^{9}$ due in part to intra-tendinous injection. PRP is defined as plasma with a platelet concentration (from 1,000000 to 2,400000 par $\mu \mathrm{L}) 3$ to 8 times higher than in blood, which promotes stem cell recruitment and directly stimulates collagen production by the tendon tenoblasts ${ }^{10}$ with proliferation and differentiation of human tenocytes in response to PRP. PRP can be directly injected into tendons to enhance local platelet concentration. Numerous in vitro ${ }^{11-13}$ and animal studies using this technique have been performed in animal models of tendon rupture or $\mathrm{T}+$ with results demonstrating improvement of clinical and histological repair ${ }^{14,15}$. Similarly, human studies have shown discordant results regarding pain reduction in different tendon locations ${ }^{16}$.

To our knowledge, no studies have been performed on an animal model to quantify the effect of PRP injection on systemic and local molecular marker as growth factors to permit tendon healing.

Recently, a descriptive laboratory study suggested, in a small number of patients $(n=25)$, that PRP intratendinous injection may trigger systemic increases basic fibroblast growth factor (bFGF), vascular endothelial growth factor (VEGF), and platelet-derived growth factor-BB (PDGF-BB) in competitive athletes with evidence that VEGF could serve as a useful molecular marker to detect athletes treated with PRP. Unfortunately, the platelet or leukocyte counts in the PRP treatment were not reported, nor were lesion size, or chronicity of the condition ${ }^{17}$.

Strong evidence that PRP might increase systemic and local growth factors to treat $T+$ in animal models is therefore not available. Like in humans, our hypothesis was that PRP increase growth factors production in an extended way. The aim of our study was thus to quantify the effect of PRP injection on systemic and local growth factors and to identify molecular markers in a rat model of patellar and Achilles $\mathrm{T}+$ treated with PRP.

\section{Materials and methods}

The procedure and animal care complied with the "Principles of animal care" formulated by the Euro- pean Union (Animal Facility Agreement 75-18-03, 2005), and animal experimentation was performed under the authorization ${ }^{* *}$ BLINDED ${ }^{\star *}$ Ministry of Agriculture.

Twenty two immunocompetent male Sprague Dawley rats (providing 88 patellar and Achilles tendons) weighing 250 to $350 \mathrm{~g}$ were used for the study. The rats were sedated before and during each manipulation with Isoflurane ${ }^{\circledR}$ ( $5 \%$ for induction and $2.5 \%$ for maintenance). Two protocols were used, one to assess the systemic molecular effect of intratendinous injection of PRP in T+ and a second one to assess local molecular effect of PRP in T+.

\section{Protocol 1 (PRP systemic effect):}

At Day 0, 10 serum samples of venous blood (jugular puncture) were made on healthy rats to take normal values of systemic biological markers for "baseline" ( $\mathrm{T}-)$. After, we induced chemical $\mathrm{T}+$ in 20 rats $(80$ patellar and Achilles tendons) by a single intratendinous injection of Type 1 Collagenase Gibco ${ }^{\mathrm{TM}}$ (250 U ie $30 \mu \mathrm{l}$, dissolved in 0.09 saline solution PROAM®) using a 29 G needle, under Ultrasound (US) guidance by a single operator. This model of $T+$ has been described in previous publications and permits one to obtain an animal model of $\mathrm{T}+$ as early as 3 days after collagenase injection and thereafter, up to 12 weeks ${ }^{18,19}$.

At day 3 , we initiated treatment using either PRP (group 1, $\mathrm{n}=7$ rats) or Physiological Serum (PS, control= group $2 \mathrm{n}=7$ rats) by the same single operator. Six rats received no injection: they allowed witnesses to the natural evolution of tendinopathy (group 3, $\mathrm{n}=6$ rats).

Treatment consisted of a single intratendinous injection, under US guidance (targeting the thickened segment of the tendon), using a $29 \mathrm{G}$ needle of either 0.1 $\mathrm{ml}$ of PRP (PRPT+) or $0.1 \mathrm{ml}$ of PS (ST+). The basic mechanisms for preparing PRP involved withdrawal of the rat's peripheral jugular blood $(3 \mathrm{ml})$ and a single 8 minute spin centrifugation $(3000 \mathrm{G})$ with no activator to obtain a final volume of PRP (visible as a yellow layer) of $1 \mathrm{ml}$. This PRP obtained had a platelet concentration equal to 3 times (mean $=1.500 .000 \pm 42.000$ ) the concentration measured in the blood as verified using a conventional cytometry method by Scil vet abc Plus ${ }^{\circledR}$. We chose this concentration as it is the lowest accepted platelet concentration for PRP without activator. Our PRP preparation was also poor in leukocytes to avoid any acute inflammatory response with catabolic effects ${ }^{20,21}$.

No specific regimen or restricted activity followed the PRP or PS injection. Figure 1 shows the 3 bottles of supernatant (Platelet Poor Plasma), PRP and red cells, after conventional cytometry method.

To compare the systemic effect of PRP, $3 \mathrm{~mL}$ of venous blood serum were collected by transjugular blood puncture (Fig. 2) for systemic biochemical assessment from each rat in each group at each time point (Tab. 1). 
The molecular systemic and local effects of intra-tendinous injection of Platelet Rich Plasma in tendinosis: preliminary results on a rat model with ELISA method

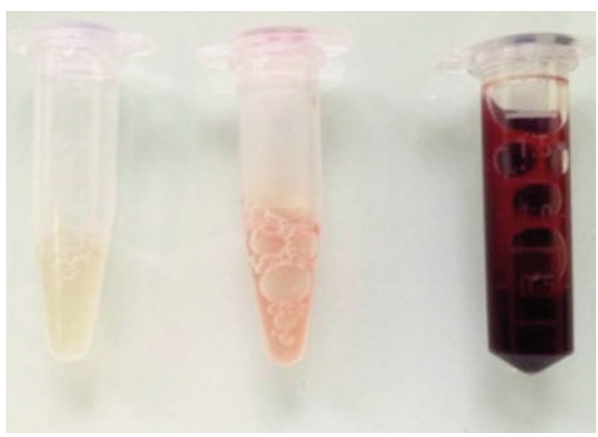

Figure 1. Presentation (from left to right) of the 3 bottles supernatant (Platelet Poor Plasma), PRP and red cells, after conventional cytometry method by Scil vet abc Plus ${ }^{\circledR}$.

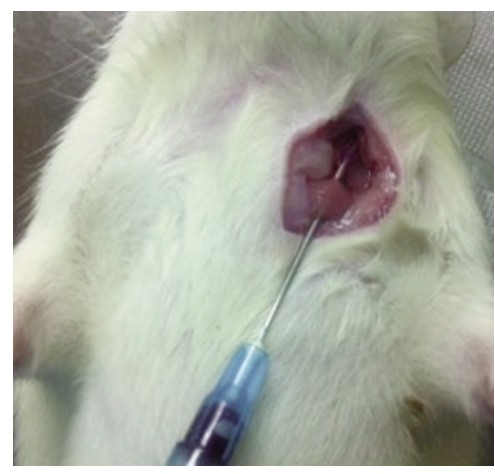

Figure 2. Recurring transjugular blood puncture $(3 \mathrm{ml})$ at day 13 for systemic biochemical assessment.

Table 1. Serum assays were performed on 3 randomly rats selected from the remaining rats not yet sacrificed. One rat per group was sacrificed at each step for the removal of tendons.

\begin{tabular}{lllllllll}
\hline & & $\mathrm{n}$ at D0 & $\mathrm{D} 3$ & $\mathrm{D} 7$ & $\mathrm{D} 13$ & $\mathrm{D} 18$ & $\mathrm{D} 25$ \\
\hline Systemic assessment (n=rats) & No injection & 6 rats & 3 & 3 & 3 & 3 & 2 \\
\cline { 2 - 8 } & PS & 7 rats & 3 & 3 & 3 & 3 & 3 \\
\cline { 2 - 8 } & PRP & 7 rats & 4 & 4 & 4 & 4 & 3 \\
\hline \multirow{2}{*}{ Local assessment (n=tendons) } & No injection & 24 tendons & 4 (1 rat) & 4 (1 rat) & 4 (1 rat) & 4 (1 rat) & 8 (2 rats) \\
\cline { 2 - 8 } & PS & 28 tendons & 4 (1 rat) & 4 (1 rat) & 4 (1 rat) & 4 (1 rat) & 12 (3 rats) \\
\cline { 2 - 8 } & PRP & 28 tendons & 4 (1 rat) & 4 (1 rat) & 4 (1 rat) & 4 (1 rat) & 12 (3 rats) \\
\hline
\end{tabular}

\section{Protocol 2 (PRP local effect):}

PRP local effect was studied by comparing 80 tendons with chemical tendinosis induction $(T+)$ injected with PRP (PRPT+, group 1, $\mathrm{n}=28$, patellar $=14$, Achilles=14), injected with PS (PST+, group 2, $n=28$, patellar $=14$, Achilles $=14)$ or not injected $(T+$, group $3, n=24$, patellar $=12$, Achilles $=12$ ).

At day $3,7,13$ and 18 , one rat (4 tendons) was sacrificed in each group. At day 25, 3 rats were sacrificed in group 1 and 2; 2 were sacrificed in group 3. Biological examination (evaluation of local biological markers) was performed on each sacrificed $T+$ rat during follow up on sample tendon.

Figure 3 shows removal of left Achilles tendon at day 25 for local biochemical assessment.

Two rats (8 tendons) served as "baseline" and have received no injection of collagenase, PRP or PS. At day 0 , they were directly sacrificed to take normal values of local biological markers in healthy patellar $(n=4$ T- $)$ and Achilles tendon $(n=4 T-)$.

All the study design was summarized in Table 1.

\section{ELISA method and data biological analysis}

Biological evaluation of blood and tendon sample used enzyme-linked immunology assay [ELISA: Bio-Plex ProTM Rat cytokine 24-plex Assay - BIO-RAD TM (order catalog $171-\mathrm{K} 1001 \mathrm{M})$ ] screening method ${ }^{17}$ with focus on:
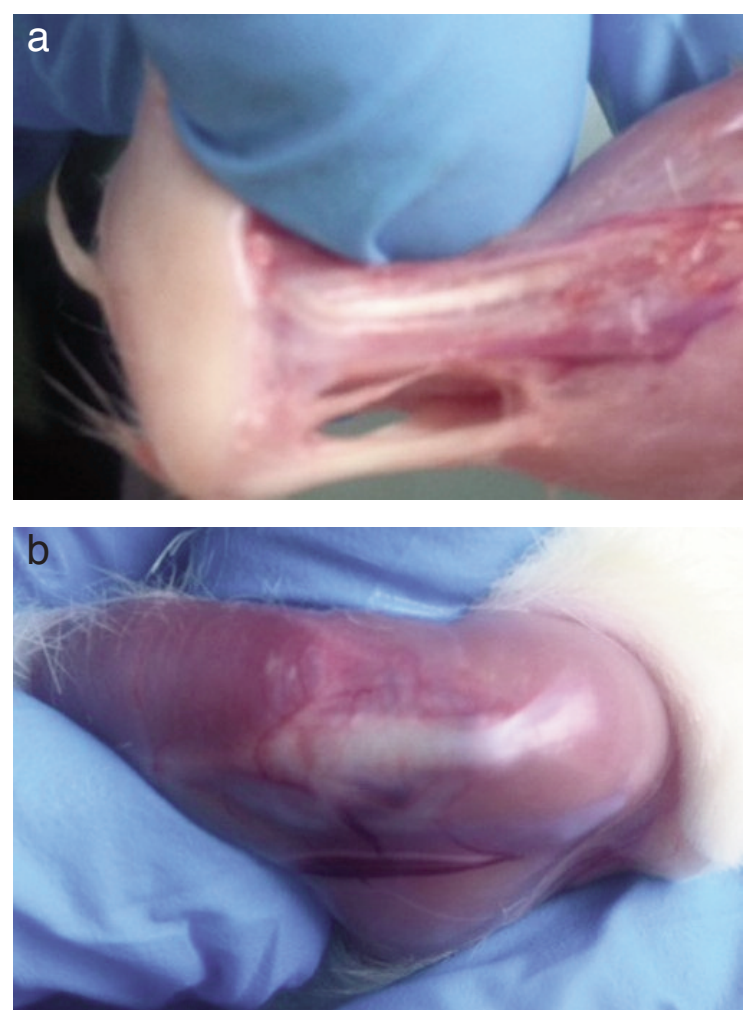

Figure 3. Removal of left Achilles tendon (a) and righ patellar tendon (b) at day 25 for local biochemical assessment. 
- Ra IL1a, 1b, 2, 4, 5, 6, 7, 10, 12, 13,17, 18.

- Ra EPO, G CSF, GM CSF.

- Ra GRO/KC, IFN-g, M-CSF.

- Ra MIP-1 ${ }^{\circ}$, MIP-3 ${ }^{\circ}$, RANTES.

- Ra TNF-a, RA VEGF.

To study the immunological markers on serum sample (protocol 1): a sample (with EDTA: Ethylenediaminetetraacetic acid) from venous blood of $2 \mathrm{ml}$ jugular level has been achieved. It has been a first centrifugation at 2,000 jets for 10 minutes. Plasma itself underwent centrifugation at 2,500 jets for 15 minutes. A tube 100 microliters was individualized for the ELISA assay.

For the study of immunological markers in tendon (protocol 2): Achilles and patellar tendons collected were weighed. Tendons were in RPMI (for 10 milligrams of tendon 100 milliliters of saline are added). The result were incubated 24 hours at $37^{\circ} \mathrm{C}$ then centrifuged at 3000 jets for 10 minutes. The supernatant was recovered and considered as sample tendon environment. These environments were individualized for assay by ELISA technique.

Operators were blinded to the status of tendons.

\section{Statistical analysis}

Statistical analysis was performed using MedCalc ${ }^{\circledR}$ software 11.0.

Comparative series were considered independent of each other. A mean value and standard deviation was calculated for each rat at each control in the 3 groups for each biological marker. To study the effects of PRP, we compared the biological systemic and local markers of PRPT+, PST+, T+ and T- at day 0 , day 3 , day 7 , day 13 , day 18 and day 25 , using paired t-tests. Then, we assessed the evolution of bi- ological systemic and local markers of PRPT+, PST+, $\mathrm{T}+$ and $\mathrm{T}$ - at day 3 , day 7 , day 13 , day 18 and day 25, using a Kruskal-Wallis test.

We considered $p<0.05$ as significant.

\section{Results}

\section{Protocol 1 (PRP systemic effect):}

During biological follow up, comparison of all serum samples of PRPT+, PST+ and T+ groups (transversal assessment) showed no significant modification of their biological markers at day $7(p=0.22)$, day 13 $(p=0.28)$, day $18(p=0.83)$ and day $25(p=0.64)$ considering each biological marker and also all subgroups confounded.

Similarly at day 0 , day 3 , day 7 , day 13 , day 18 and day 25 (longitudinal assessment), ELISA showed no significant modification of each biological markers in each group $\mathrm{T}-$, PRPT+, PST+ and $\mathrm{T}+(\mathrm{p}>0.73)$.

Table 2 summarizes mean and standard deviation of each biological systemic sample for PRPT+, PST+ Tand $T+$ animals at day 0 , day 3 , day 7 , day 13 , day 18 and day 25.

\section{Protocol 2 (PRP local effect):}

During biological follow up, comparison of immunological sample tendon markers of PRPT+, PST+ and $T+$ groups (transversal assessment) showed no significant modification of their biological markers at day $7(p=0.2)$, day $13(p=0.58)$, day $18(p=0.4)$ and day $25(p=0.16)$ considering each biological marker and also all subgroups confounded.

Table 2. Mean and standard deviation of systemic biological markers at day 0, 3, 6, 13, 18 and 25.

\begin{tabular}{|c|c|c|c|c|c|c|c|c|c|c|c|c|c|c|c|c|c|c|c|c|}
\hline \multicolumn{3}{|c|}{ Biological Marker } & \multicolumn{6}{|c|}{ Placebo Group } & \multicolumn{6}{|c|}{ P Serum Group } & \multicolumn{6}{|c|}{ PRP Group } \\
\hline & \multicolumn{2}{|c|}{ Do Baseline } & \multirow{2}{*}{\begin{tabular}{|l} 
D13 \\
Mean
\end{tabular}} & \multirow[b]{2}{*}{$S D$} & \multirow{2}{*}{\begin{tabular}{|l|} 
D18 \\
Mean \\
\end{tabular}} & \multirow[b]{2}{*}{$S D$} & \multirow{2}{*}{\begin{tabular}{|l} 
D25 \\
Mean
\end{tabular}} & \multirow[b]{2}{*}{ SD } & \multirow{2}{*}{\begin{tabular}{|l|} 
D13 \\
Mean
\end{tabular}} & \multirow[b]{2}{*}{ SD } & \multirow{2}{*}{\begin{tabular}{|l} 
D18 \\
Mean
\end{tabular}} & \multirow[b]{2}{*}{ SD } & \multirow{2}{*}{\begin{tabular}{|l|} 
D25 \\
Mean
\end{tabular}} & \multirow[b]{2}{*}{ SD } & \multirow{2}{*}{\begin{tabular}{|l} 
D13 \\
Mean
\end{tabular}} & \multirow[b]{2}{*}{ SD } & \multirow{2}{*}{\begin{tabular}{|l} 
D18 \\
Mean
\end{tabular}} & \multirow[b]{2}{*}{ SD } & \multirow{2}{*}{\begin{tabular}{|l|} 
D25 \\
Mean
\end{tabular}} & \multirow[b]{2}{*}{ SD } \\
\hline & Mean & SD & & & & & & & & & & & & & & & & & & \\
\hline EPO & 133.84 & 252,84 & 1447.16 & 1681.8 & 733.15 & 663.73 & 253.23 & 390.87 & 614.72 & 484.2 & 532.86 & 360.5 & 60.58 & 25.67 & 344.17 & 339.0 & 894.27 & 535.7 & 131.75 & 260.59 \\
\hline TNF alpha & 1.54 & 3,4 & 14.25 & 7.1 & 22.58 & 23.73 & 5.59 & 5.66 & 5.11 & 6.2 & 27.51 & 22.6 & 1.85 & 0.61 & 5.18 & 5.8 & 9.01 & 2.5 & 5.13 & 2.66 \\
\hline GM-CSF & 2.88 & 1,2 & 14.75 & 6.3 & 10.14 & 4.37 & 10.35 & 10.08 & 11.84 & 13.4 & 44.51 & 22.2 & 5.08 & 4.12 & 6.65 & 6.6 & 13.98 & 9.0 & 4.94 & 3.46 \\
\hline VEGF & 5.42 & 2,24 & 5.05 & 1.2 & 4.46 & 0.79 & 4.88 & 1.45 & 3.67 & 3.1 & 13.48 & 5.5 & 2.75 & 2.21 & 2.32 & 2.4 & 4.44 & 0.3 & 3.97 & 1.68 \\
\hline GRO/KC & 15.30 & 6,58 & 46.23 & 44.9 & 27.15 & 24.14 & 40.82 & 37.99 & 22.17 & 33.6 & 52.48 & 36.8 & 17.02 & 18.15 & 9.57 & 3.9 & 17.44 & 20.6 & 6.76 & 7.55 \\
\hline IL1-a & 8.59 & 6,22 & 26.06 & 24.5 & 7.67 & 0.82 & 3.89 & 4.02 & 8.60 & 10.9 & 78.96 & 41.0 & 0.74 & 0.57 & 9.56 & 6.0 & 6.65 & 7.1 & 8.45 & 1.55 \\
\hline IL1-b & 12.54 & 4,2 & 32.05 & 5.9 & 26.96 & 6.60 & 21.26 & 20.20 & 25.43 & 25.4 & 428.00 & 280.3 & 19.03 & 16.82 & 17.98 & 20.7 & 36.03 & 18.5 & 18.80 & 8.56 \\
\hline IL2 & 29.80 & 14,2 & 42.66 & 34.4 & 17.52 & 11.57 & 13.06 & 6.02 & 24.07 & 30.4 & 40.51 & 34.3 & 2.53 & 0.12 & 11.67 & 16.8 & 16.44 & 14.1 & 24.11 & 2.37 \\
\hline IL4 & 4.79 & 1,3 & 3.65 & 0.4 & 2.66 & 1.09 & 3.26 & 2.91 & 7.20 & 5.5 & 45.84 & 30.1 & 1.35 & 1.24 & 3.64 & 3.0 & 4.00 & 2.6 & 2.59 & 0.64 \\
\hline IL5 & 9.48 & 2,04 & 49.91 & 6.8 & 38.40 & 6.55 & 44.14 & 22.32 & 31.08 & 27.9 & 167.81 & 63.2 & 21.73 & 8.51 & 21.27 & 22.4 & 41.49 & 21.0 & 32.60 & 9.98 \\
\hline IL6 & 73.14 & 10,23 & 113.89 & 118.7 & 17.26 & 11.44 & 11.93 & 15.26 & 25.18 & 37.7 & 429.10 & 237.8 & 3.52 & 2.11 & 32.60 & 57.9 & 24.24 & 35.3 & 36.76 & 14.37 \\
\hline IL7 & 144.93 & 28,24 & 204.11 & 250.3 & 71.56 & 85.62 & 167.66 & 66.78 & 59.02 & 93.9 & 273.08 & 211.2 & 93.42 & 40.73 & 143.77 & 265.8 & 83.18 & 57.1 & 136.90 & 92.94 \\
\hline IL10 & 11.95 & 4,7 & 47.26 & 10.0 & 38.28 & 15.95 & 43.44 & 37.21 & 36.87 & 33.8 & 27.51 & 9.7 & 22.61 & 19.38 & 23.66 & 26.2 & 46.64 & 20.1 & 32.80 & 11.93 \\
\hline IL12p70 & 5.77 & 1,25 & 6.35 & 2.1 & 3.97 & 1.31 & 5.08 & 4.58 & 4.29 & 5.5 & 39.45 & 32.2 & 1.30 & 1.86 & 2.28 & 2.4 & 8.66 & 4.6 & 5.46 & 2.98 \\
\hline IL13 & 5.77 & 2,33 & 16.93 & 6.3 & 8.83 & 5.03 & 9.88 & 9.46 & 10.69 & 11.8 & 31.99 & 26.6 & 5.44 & 6.17 & 5.43 & 4.8 & 14.70 & 11.0 & 5.20 & 4.72 \\
\hline LL17a & 5.20 & 1,2 & 4.24 & 2.4 & 1.81 & 1.36 & 2.30 & 2.20 & 2.64 & 3.3 & 31.19 & 17.0 & 0.64 & 0.74 & 0.82 & 0.8 & 2.71 & 2.6 & 3.08 & 1.07 \\
\hline IL18 & 683.73 & 125,65 & 6114.29 & 6378.8 & 3837.83 & 3261.36 & 1880.22 & 1715.84 & 2234.72 & 1922.9 & 1441.57 & 875.6 & 1192.90 & 1527.41 & 1159.97 & 1170.1 & 3735.13 & 2379.8 & 2613.13 & 1224.72 \\
\hline M-CSF & 121.68 & 24,5 & 169.21 & 34.4 & 185.82 & 16.30 & 135.25 & 7.07 & 149.77 & 123.1 & 157.84 & 21.2 & 131.69 & 26.81 & 91.76 & 95.1 & 193.53 & 26.0 & 153.72 & 69.04 \\
\hline MIP3a & 9.91 & $1, \mathbf{1}$ & 8.13 & 1.3 & 4.87 & 1.06 & 5.99 & 4.38 & 4.63 & 3.9 & 29.50 & 12.7 & 5.58 & 4.68 & 3.23 & 3.2 & 8.54 & 2.9 & 4.32 & 2.71 \\
\hline Rantes & 28.61 & 9,4 & 63.68 & 11.4 & 27.33 & 8.93 & 41.08 & 32.78 & 33.50 & 40.2 & 25.47 & 4.7 & 21.61 & 14.07 & 11.78 & 7.2 & 31.74 & 24.1 & 14.51 & 11.25 \\
\hline
\end{tabular}


The molecular systemic and local effects of intra-tendinous injection of Platelet Rich Plasma in tendinosis: preliminary results on a rat model with ELISA method

Similarly at day 0 , day 3 , day 7 , day 13 , day 18 and day 25 (longitudinal assessment), ELISA showed no significant modification of biological markers in each group T-, PRPT+, PST+ and T+ ( $>0.14)$.

Table 3 summarizes mean and standard deviation of each immunological sample tendon markers for PRPT+, PST+ T- and T+ animals at day 0 , day 3 , day 7 , day 13 , day 18 and day 25 whereas graphs 1 and 2 focus about main cytokine systemic and local evolution in the different groups.

Table 3. Mean and standard deviation of local biological markers at day 0, 3, 6, 13, 18 and 25.

\begin{tabular}{|c|c|c|c|c|c|c|c|c|c|c|c|c|c|c|c|c|c|c|c|c|}
\hline \multirow{2}{*}{$\begin{array}{l}\text { Biological } \\
\text { Marker }\end{array}$} & \multirow[b]{2}{*}{ Baseline } & & \multicolumn{6}{|c|}{ Placebo Group } & \multicolumn{6}{|c|}{ Serum Group } & \multicolumn{6}{|l|}{ P'RP Group } \\
\hline & & & D13 & & D18 & & D25 & & D13 & & D18 & & D25 & & D13 & & D18 & & D25 & \\
\hline & Mean & SD & Mean & SD & Mean & SD & Mean & SD & Mean & SD & Mean & SD & Mean & SD & Mean & SD & Mean & SD & Mean & SD \\
\hline EPO & 166.320 & 143.841 & 369.807 & 527.974 & 215.898 & 225.915 & 333.893 & 432.830 & 864.090 & 507.957 & 183.728 & 128.778 & 218.405 & 236.876 & 240.385 & 314.332 & 175.748 & 124.152 & 452.474 & 640.157 \\
\hline TNF alpha & 9.152 & 4.662 & 26.318 & 23.592 & 19.898 & 4.979 & 13.125 & 4.864 & 21.090 & 19.223 & 14.725 & 6.453 & 9.774 & 4.510 & 16.560 & 18.245 & 13.125 & 4.864 & 16.709 & 17.548 \\
\hline GMCSF & 3.410 & NM & 7.170 & NM & 30.640 & 35.081 & 13.845 & 23.498 & 3.880 & 0.396 & 29.290 & 39.471 & 4.865 & 6.205 & 12.960 & 17.423 & 60.505 & 30.314 & 6.092 & 5.385 \\
\hline VEGF & 1226.721 & 972.574 & 2053.938 & 764.059 & 2434.140 & 192.399 & 1379.693 & 917.481 & 1670.660 & 1125.099 & \begin{tabular}{|l|l|}
1894.103 \\
\end{tabular} & 849.558 & 1291.697 & 7958.703 & 2247.333 & 566.764 & 1764.808 & 8626.159 & 979.144 & 774.459 \\
\hline GRO/KC & 1808.886 & 2116.339 & 3521.893 & |887.918 & 3499.980 & NM & 3366.344 & 4. 466.873 & 3395.210 & 688.430 & 3290.083 & 257.434 & 3336.338 & 8622.182 & 3491.687 & 475.942 & 3621.025 & 5475.805 & 2876.287 & $\begin{array}{ll}7118.185 \\
\end{array}$ \\
\hline L1-a & 7.490 & 6.153 & 10.580 & 10.761 & 23.613 & 12.392 & 9.238 & 6.268 & 10.233 & 2.015 & 13.343 & 14.880 & 10.361 & 12.602 & 12.430 & 8.292 & 52.163 & 70.910 & 5.635 & 4.037 \\
\hline L1-b & NM & NM & 44.265 & 36.597 & 63.090 & 55.824 & 23.163 & 33.954 & 32.500 & 20.597 & 105.480 & NM & 39.995 & 33.568 & 36.648 & 21.017 & 6.510 & 1.103 & 10.455 & 6.747 \\
\hline MCP-1 & 2431.781 & 3515.834 & 9329.505 & 54330.581 & 9329.595 & 1608.891 & 1) 6492.871 & 14080.019 & 6402.095 & 5434.827 & 8360.923 & 2095.054 & 5400.172 & $2 \mid 4114.211$ & 11684.44 & 2550.006 & 57306.373 & 34465.130 & $\begin{array}{l}0 \\
0\end{array}$ & 34002.724 \\
\hline IL2 & 6.008 & 4.676 & 29.640 & 24.025 & 28.565 & 13.800 & 18.740 & 11.306 & 22.555 & 13.907 & 25.648 & 19.926 & 14.222 & 7.670 & 21.308 & 16.007 & 16.618 & 7.510 & 13.905 & 9.583 \\
\hline IL4 & 0.693 & 0.504 & 4.430 & 3.324 & 3.743 & 2.292 & 2.256 & 1.697 & 3.310 & 2.746 & 3.360 & 2.902 & 1.887 & 0.810 & 3.663 & 2.597 & 2.265 & 1.510 & 1.024 & 0.774 \\
\hline 115 & 18.053 & 12.372 & 41.170 & 10.808 & 46.855 & 11.188 & 33.754 & 13.821 & 39.030 & 16.682 & 36.923 & 13.202 & 31.208 & 16.402 & 49.240 & 12.060 & 36.300 & 13.939 & 28.321 & 16.714 \\
\hline IL6 & 3382.428 & 1593.634 & 44473.863 & 31861.914 & 5177.540 & 40.868 & 4972.757 & 7224.944 & 4794.693 & 428.603 & 5189.320 & 113.297 & 4410.127 & \begin{tabular}{l|l|l}
1231.003 \\
\end{tabular} & 35124.673 & 226.304 & 4995.120 & 292.992 & 3992.605 & 1443.205 \\
\hline 117 & 50.483 & 55.732 & 181.555 & 290.315 & 136.763 & 73.566 & 105.284 & 111.574 & 205.023 & 244.115 & 89.643 & 41.955 & 82.333 & 67.999 & 117.728 & 149.741 & 62.890 & 42.799 & 149.139 & 220.943 \\
\hline IL10 & 71.000 & 76.712 & 927.505 & 900.022 & 7648.535 & 13126.66 & 335.677 & 283.176 & 1459.640 & 1345.801 & 1512.225 & 2436.620 & 245.594 & 336.567 & 365.223 & 365.903 & 2811.078 & 85031.938 & $\begin{array}{l}8 \\
883.197\end{array}$ & 504.194 \\
\hline |L12p70 & 4.890 & 3.336 & 12.510 & 14.439 & 9.387 & 10.994 & 5.796 & 4.003 & NM & NM & 15.420 & 16.292 & 2.273 & 3.697 & 8.165 & 6.371 & 1.190 & 1.344 & 5.338 & 3.569 \\
\hline IL13 & NM & NM & NM & NM & 9.387 & 10.994 & 5.796 & 4.003 & 1.500 & NM & 2.810 & NM & 0.580 & 0.806 & 0.943 & 0.460 & 0.380 & NM & 0.500 & NM \\
\hline IL17a & 10.470 & NM & 19.783 & 20.376 & 23.820 & 5.218 & 15.042 & 4.075 & 56.140 & 74.882 & 10.110 & 10.493 & 3.790 & 0.953 & 11.453 & 10.621 & NM & NM & 9.970 & 4.158 \\
\hline IL18 & 12.280 & NM & 60.290 & 75.356 & 47.355 & 18.037 & 54.282 & 41.625 & 69.780 & 62.398 & 19.573 & 16.829 & 16.143 & 15.020 & 47.060 & 43.489 & 24.173 & 12.463 & 47.342 & 46.832 \\
\hline MCSF & 39.010 & 24.980 & 44.900 & 19.554 & 73.520 & 27.516 & 55.278 & 36.842 & 34.725 & 8.391 & 93.650 & 52.707 & 44.063 & 27.968 & 65.565 & 30.374 & 81.623 & 31.070 & 48.152 & 36.039 \\
\hline MIP-3a & 6.313 & 3.483 & 95.640 & 114.332 & 235.645 & 277.131 & 31.935 & 46.401 & 66.163 & 46.940 & 232.118 & 91.640 & 18.723 & 31.050 & 49.505 & 58.016 & 292.895 & 552.473 & 9.694 & 9.038 \\
\hline Rantes & 23.380 & 10.340 & 53.480 & 35.412 & 45.048 & 14.652 & 32.351 & 9.764 & 46.618 & 19.291 & 38.785 & 29.174 & 30.537 & 5.891 & 41.263 & 9.520 & 78.418 & 104.966 & 32.801 & 10.889 \\
\hline
\end{tabular}
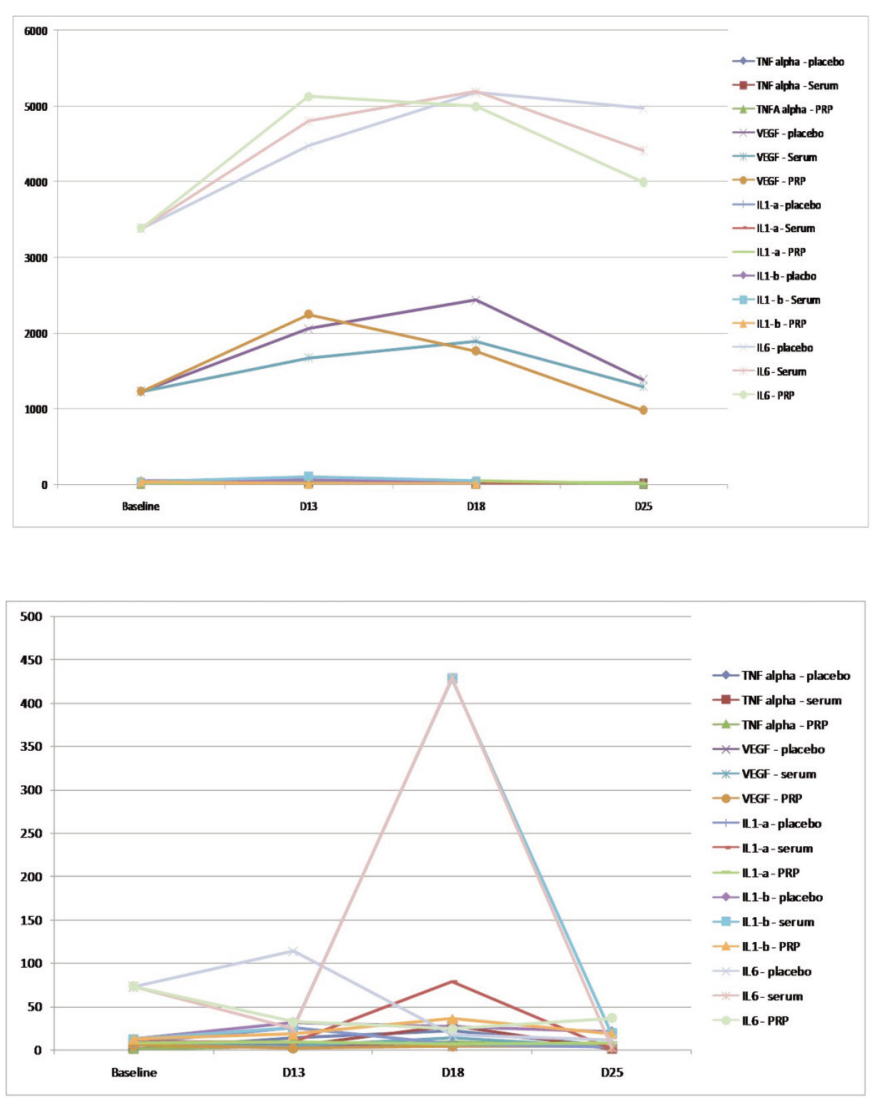

Graph 1. Systemic main cytokines evolution in PRPT+, PST+ and T+ groups (transversal assessment): TNF alpha, VEGF, IL-1a, IL-1B, and IL-6.

Graph 2. Local main cytokines evolution in PRPT+, $\mathrm{PST}+$ and $\mathrm{T}+$ groups (transversal assessment): TNF alpha, VEGF, IL-1a, IL-1B, and IL-6. 


\section{Discussion}

Our study strongly suggests that a single intratendinous US-guided injection of PRP in Achilles and patellar T+ doesn't increase biological markers such as growth factors compared to a control group in midterm and long-term follow-up. These results are potentially important as, to our knowledge, there has been no study demonstrating the immunological effects of PRP, early in the natural evolution of tendinopathy, before tendon rupture and before the onset of chronic pain.

Reports assessing autologous blood, PRP or varicose vein-sclerosing drugs, injected in different sites (intra, peritendinous) under different conditions (clinically-guided, imaging-guided), in heterogeneous populations, including patients with $\mathrm{T}+$ and patients with rupture, with no long term follow-up and no histological examination ${ }^{9,22,23}$ have provided contradictory results. Thus, there is no clear conclusion regarding the curative effect of PRP in T+.

By recently assessing PRP in a rat model [with fixed platelet concentration in PRP (x3) and no adjuvant], with a systematic clinical and US follow-up and histological examination, we have previously provide strong evidence that PRP might be a useful strategy to treat $T+{ }^{15}$. In this study, we used the same setup, animal model, and controlled PRP preparation protocol as described in our pre-clinical model. Based on these results and the potential healing of PRP to stimulate thrombus in tendon as described above, our next step was therefore to assess, after clinical, US and histological evaluation, the biological potential effect PRP to increase growth factor concentration as recently investigated and described in human ${ }^{17}$.

Indeed, in literature, intratendinous PRP injection, by locally providing important concentration of active growth factors (PDGF, TGF- $\beta$, VEGF...), might promote stem cell recruitment and fibroblast collagen production, and therefore stimulate tendon cicatrization ${ }^{10}$. Moreover, in recent series on human model, Wasterlain AS et al. assayed 6 growth factors by ELISA method in 25 patients before and after intratendinous injection of PRP: human growth hormone (hGH), insulin-like growth factor-1 (IGF -1), insulin-like growth factor binding protein- 3 (IGFBP -3), basic fibroblast growth factor (bFGF or FGF -2), vascular endothelial growth factor (VEGF) and platelet-derived growth factor-BB (PDGF- BB). They showed a significant increase in blood of several growth factors, particularly VEGF which would be the best serum marker of PRP therapy. However, this study, with multiple blood puncture near to the injection of PRP time, only followed the athletes during $96 \mathrm{~h}$ (6 noncash between 0.25 and $96 \mathrm{~h}$ ), with no food or any type of physical exercise 3 hours before each serum sample. His aim was rather to know the short-term effects of injection of PRP for assessing growth factors in the doping. Indeed, PRP treats sports 86,000 athletes in the United States each year and is not considered as doping substance, while growth factors are banned by the World Anti-Doping Agency (WADA).
In our animal experience, the first serum assays were beginning $72 \mathrm{~h}$ post PRP injection with a long term follow up of 25 days without limitation in terms of food or exercise for animals, in order to correlate possible changes in serum phases of tendon repair, but even early assays at day 3 showed no difference. In another animal study performed on horses, McCarrel TM et al. ${ }^{21}$ demonstrate a lower level of expression of interleukin 1 beta and TNF alpha in tendons treated with a low concentration of leucocytes in PRP compared to intermediate and high levels. This could explain in part our results concerning IL 1 and TNF alpha, in comparison with Wasterlain et al. in which the platelet or leukocyte counts in the PRP treatment were not reported. Moreover, we didn't find any significant changes in the evaluated biomarkers after collagenase injection during the natural evolution of $\mathrm{T}+$ in the untreated group. We also highlight an interesting to know the important inter-individual variation for all interleukins and growth factors at day 0 (baseline) in the physiological state, without inducing element tendinopathy. Indeed, we observed very different values, including VEGF ranging from non-detectable to important value (Tab. 2). These very different inter-individual perspective core values could be an answer as to the ineffectiveness of PRP in some individuals. A dosing study of physiological values of growth factors and interleukins humans (in a non-patient population) to search for possible differences might be interesting to explain some differences in efficiency, according to the Authors, protocols and series.

Our in vitro study suffers from three biases: first, we measured prostaglandins, interleukins and growth factors directly in the serum using ELISA, instead of the more commonly used method that indirectly measured RNA with RT-PCR ${ }^{24}$. We choose the ELISA method as we had been using it since many years in our laboratory where the method was validated with internal controls and used in the work of several publications ${ }^{25,26}$. Second, twenty-two rats were included in our study but due to the iterative sacrifices to allow the collection of tendons during follow-up, only 3 rats in the group without injection as well as in the group treated with PS and only 4 in the PRP group were taken at each step. Although this population was sufficient for statistical calculation, additional studies including a larger number of rats will be needed in the future to confirm our preliminary results. Third, we did not focus on short term biological effect of PRP and an earlier measurement should be performed in future studies. Such results necessitate however additional studies before the protocol can be applied to human patients ${ }^{27}$.

\section{Conclusion}

Our study suggests that a mono-injection of PRP in tendinosis using a controlled concentration of platelets and leukocytes doesn't increase biological markers such as growth factor in rats in mid-term and longterm follow-up, according to ELISA criteria. Biological 
The molecular systemic and local effects of intra-tendinous injection of Platelet Rich Plasma in tendinosis: preliminary results on a rat model with ELISA method

studies, prospectively comparing PRP to placebo control group should be initiated in the future.

\section{References}

1. Goel DP, Chan D, Watson K, Mohtadi N. Safety and hospital costs of Achilles tendon surgery: the serendipitous impact of a randomized clinical trial. Can J Surg. 2009;52(6):467-472.

2. Cotten A. Imagerie Musculo-squelettique, Pathologies locorégionales, Tome 2. Edition Masson, Paris. 3-133.

3. Sharma P, Maffulli N. Tendon injury and tendinopathy: healing and repair. J Bone Joint Surg Am. 2005;87(1):187-202.

4. Housner JA, Jacobson JA, Misko R. Sonographically guided percutaneous needle tenotomy for the treatment of chronic tendinosis. J Ultrasound Med. 2009;28(9):1187-1192.

5. Rabago D, Best TM, Zgierska AE, Zeisig E, Ryan M, Crane D. A systematic review of four injection therapies for lateral epicondylosis: prolotherapy, polidocanol, whole blood and platelet-rich plasma. Br J Sports Med. 2009;43(7):471-481.

6. Park E-J, Kim E-S, Weber H-P, Wright RF, Mooney DJ. Improved bone healing by angiogenic factor-enriched plateletrich plasma and its synergistic enhancement by bone morphogenetic protein-2. Int J Oral Maxillofac Implants. 2008;23(5): 818-826.

7. De Jonge S, De Vos RJ, Weir A, et al. One-year follow-up of platelet-rich plasma treatment in chronic Achilles tendinopathy: a double-blind randomized placebo-controlled trial. Am J Sports Med. 2011;39(8):1623-1629.

8. Dohan Ehrenfest DM, Andia I, Zumstein MA, Zhang CQ, Pinto $\mathrm{NR}$, Bielecki T. Classification of platelet concentrates (PlateletRich Plasma-PRP, Platelet-Rich Fibrin-PRF) for topical and infiltrative use in orthopedic and sports medicine: current consensus, clinical implications and perspectives. MLTJ. 2014; 8;4(1):3-9.

9. Coombes BK, Bisset L, Vicenzino B. Efficacy and safety of corticosteroid injections and other injections for management of tendinopathy: a systematic review of randomised controlled trials. Lancet. 2010;376(9754):1751-1767.

10. Tohidnezhad M, Varoga D, Wruck CJ, et al. Platelet-released growth factors can accelerate tenocyte proliferation and activate the anti-oxidant response element. Histochem. Cell Biol. 2011;135(5):453-460.

11. Carofino B, Chowaniec DM, McCarthy MB, et al. Corticosteroids and local anesthetics decrease positive effects of platelet-rich plasma: an in vitro study on human tendon cells. Arthroscopy. 2012;28(5):711-719.

12. Geburek F, Stadler P. Regenerative therapy for tendon and ligament disorders in horses. Terminology, production, biologic potential and in vitro effects. Tierarztl Prax Ausg G Grosstiere Nutztiere. 2011;39(6):373-383.

13. Jo CH, Kim JE, Yoon KS, Shin S. Platelet-rich plasma stimu- lates cell proliferation and enhances matrix gene expression and synthesis in tenocytes from human rotator cuff tendons with degenerative tears. Am J Sports Med. 2012;40(5):10351045.

14. Parafioriti A, Armiraglio E, Del Bianco S, Tibalt E, Oliva F, Berardi AC. Single injection of platelet-rich plasma in a rat Achilles tendon tear model. MLTJ. 2011;1(2):41-47.

15. Zhang J, Wang JH. PRP treatment effects on degenerative tendinopathy - an in vitro model study. MLTJ. 2014;4(1):1017.

16. Dallaudière $B$, Lempicki $M$, Pesquer $L$, et al. Efficacy of intratendinous injection of platelet-rich plasma in treating tendinosis: comprehensive assessment of a rat model. Eur Radiol. 2013;23(10):2830-2837.

17. Wasterlain AS, Braun HJ, Harris AH, Kim HJ, Dragoo JL. The systemic effects of platelet-rich plasma injection. Am J Sports Med. 2013;41(1):186-193.

18. Lake SP, Ansorge HL, Soslowsky LJ. Animal models of tendinopathy. Disabil Rehabil. 2008;30(20-22):1530-1541.

19. Dallaudière $B$, Lempicki $M$, Pesquer $L$, et al. Acceleration of tendon healing using US guided intratendinous injection of bevacizumab: first pre-clinical study on a murine model.Eur $\mathrm{J} \mathrm{Ra-}$ diol. 2013;82(12):e823-828.

20. Dragoo JL, Braun HJ, Durham JL, et al. Comparison of the acute inflammatory response of two commercial platelet-rich plasma systems in healthy rabbit tendons. Am J Sports Med. 2012;40(6):1274-1281.

21. McCarrel TM, Minas T, Fortier LA. Optimization of leukocyte concentration in platelet-rich plasma for the treatment of tendinopathy. J Bone Joint Surg Am. 2012;94(19):e143(1-8).

22. Dallaudière $B$, Pesquer $L$, Meyer $P$, et al. Intratendinous injection of platelet-rich plasma under US guidance to treat tendinopathy: a long-term pilot study. J Vasc Interv Radiol. 2014;25(5):717-723.

23. Dallaudière B, Meyer $P$, Hummel V, et al. Efficacy of second intra-tendinous platelet-rich-plasma injection in case of incomplete response of the first injection: three-year follow up experience. Diagn Interv Imaging. 2013;94(9):871-877.

24. Alfredson $\mathrm{H}$, Lorentzon $\mathrm{M}$, Bäckman $\mathrm{S}$, Bäckman $\mathrm{A}$, Lerner $\mathrm{UH}$. cDNA-arrays and real-time quantitative PCR techniques in the investigation of chronic Achilles tendinosis. J Orthop Res. 2003;21(6):970-975.

25. Guedj K, Khallou-Laschet J, Clement M, et al. Inflammatory micro-environmental cues of human atherothrombotic arteries confer to vascular smooth muscle cells the capacity to trigger lymphoid neogenesis. PLoS One. 2014;9(12):e116295.

26. Clement M, Guedj K, Andreata F, et al. Control of the T Follicular Helper-Germinal Center B-Cell Axis by CD8+ Regulatory T Cells Limits Atherosclerosis and Tertiary Lymphoid Organ Development. Circulation. 2015;131(6):560-570.

27. Padulo J, Oliva F, Frizziero A, Maffulli N. Muscles, Ligaments and Tendons Journal. Basic principles and recommendations in clinical and field science research. MLTJ. 2013;4:250-252. 